\title{
Analysis and Prediction of Thermal Resistance for High Power LED Using GA-SVR
}

\author{
Zeng Dehuai ${ }^{1,3}$, Liu Yuan ${ }^{1}$, Li Li ${ }^{1}$, Yu Degui ${ }^{1}$, Xu Gang ${ }^{1,2, ~ *}$ \\ ${ }^{1}$ College of Mechatronics and Control Engineering, Shenzhen University, Shenzhen, 518060 \\ ${ }^{2}$ Key Laboratory of Advanced Optical Precision Manufacturing Technology of Guangdong Higher \\ Education Institutes, Shenzhen University, Shenzhen, 518060, China \\ ${ }^{3}$ School of mechanical and automotive engineering, South China University of Technology, \\ Guangzhou, 510640
}

*Corresponding author email: xgrobot@126.com

Keywords: High power LED, Junction temperature, Thermal resistance, SVR

\begin{abstract}
With the development of high power LED technology, junction temperature as a key factor constrains the performance and the service life of LED, and the main parameter of junction temperature is thermal resistance. Therefore, how to measure the thermal resistance of high power LED quickly and accurately plays an important part in improving the performance and the service life of LED. In this paper the accurate and fast measurement equipment was applied to study the thermal characteristics of high power LED. The forward-voltage based method was conducted to measure the junction temperature of high power. Then, support vector regression (SVR) combined with genetic algorithm (GA) for its parameter optimization, was proposed to establish a model to predict the thermal resistance of high power LED. The prediction performance of GA-SVR was compared with those of BPNN model. The result demonstrated that the estimated errors GA-SVR models, such as Mean Absolute Relative Error (MARE) and Root Mean Squared Errors (RMSE), all are smaller than those achieved by the BPNN applying identical samples.
\end{abstract}

\section{Introduction}

Energy crisis, global warming, energy saving and emission reduction have become some of the most concerned topics in the world. As a new generation green solid-state light source, high power Light Emitting Diode (LED) has become a promising candidate for the replacement of incandescent and fluorescent lamps in general lighting applications. due to its distinctive advantages such as high luminous efficiency, energy saving, long lifetime, and being environment-friendly. However, the photoelectric efficiency of high power LED is just only $10 \% \sim 30 \%$, in other words, almost $70 \% \sim 90 \%$ electrons change into heat [1].

the high-power LEDs used for the general lighting is the most challenge task. It is because that, as driving LEDs with high power, the heat generated in the active region has to be dissipated effectively. If the heat generated in the LED chips cannot be dissipated rapidly, the generated heat would accumulate in the LED package and cause the junction temperature of LED chips increasing. As a result, the internal quantum efficiency of LEDs will drop significantly. Also, the operational lifetime and reliability of the high power LED may be significantly reduced[2]. Thermal resistance affects light output performance and lifetime of LEDs. Accordingly, measuring the thermal resistance of LED accurately is very important for the reliability and application of LEDs.

There are several methods to measure the thermal resistance of LEDs, such as infrared thermograph method, photo thermal resistance scanning method, optical power method, emission-peak-shift method [3] and forward voltage method [4,5]. The forward voltage method due to the advantages of high accuracy, high speed and nondestructive becomes the international standard method. PN junction is the tested object and temperature sensor at the meantime. It can eliminate error from the added temperature sensor. 
In this study, we propose real-value genetic algorithms (RGA) to determine free parameters of SVR, known as GA-SVR, which optimizes all SVR's parameters simultaneously from the training data. Then, the high power LEDS thermal resistance was predicted by means of the proposed GA-SVR hybrid model. In Section 2, we provide a detailed description of SVR and GA-SVR models. In Section 3, we describe the data source and experimental settings. Then the results of RGA have been analyzed and the parameters for SVR have been optimized, followed by the discussion of the experimental results in Section 4. In addition, we also compare our method with that of BPNN. Finally, we present our conclusions in Section 5.

\section{Regression arithmetic of support vector machine}

SVR is to map the input data $x$ into a higher dimensional feature space through a nonlinear mapping $F$ and then a linear regression problem is obtained and solved in this feature space. Here, given a set of data $T=\left\{\left(x_{1}, y_{1}\right), \cdots,\left(x_{i}, y_{i}\right), \cdots,\left(x_{l}, y_{l}\right)\right\}$, where $x_{i} \in R^{n}$ is an arbitrary data point and $y_{i} \in R$ denotes the corresponding output value, the SVM regression function can be formulated as

$$
y=\omega \cdot \varphi(x)+b
$$

where $\varphi(\cdot)$ denotes the non-linear mapping function, $\omega$ denotes the weight vector and $\mathrm{b}$ denotes the bias term. $\varepsilon$-SVR is to find a function $y$ that has at most $\varepsilon$ deviation from the actually obtained targets $d_{i}$ for all the training data and at the same time is as flat as possible. We do not care about errors as long as they are less than $\varepsilon$, but will not accept any deviation larger than this. Flatness in this case means to reduce the model complexity, so that we can write this problem as a convex optimization problem:

$$
\operatorname{Min} R(y)=C \frac{1}{N} \sum_{i=1}^{n} L_{\varepsilon}\left(d_{i}, y_{i}\right)+\frac{1}{2}\|\omega\|^{2}
$$

It is known that the regression estimation function is the one that minimizes (2) with the following $\varepsilon$-insensitive loss function, defined as:

$$
L_{\varepsilon}\left(d_{i}, y_{i}\right)=\left\{\begin{array}{cc}
\left|d_{i}-y_{i}\right|-\varepsilon, & \left|d_{i}-y_{i}\right|>\varepsilon \\
0, & \text { otherwise }
\end{array}\right.
$$

where both $C$ and $\varepsilon$ are user-determined parameters; $d_{i}$ denotes the actual value at period $i ; y_{i}$ represents the forecasting value at period $i$. Additionally, the first term in (2) denotes the empirical error. The second term in (2) represents the function flatness. The $C$ is used as the trade-off between the empirical risk and the model flatness. Sometimes, this may not be the case, or we also may want to allow for some errors. Two positive slack variables $\xi_{i}$ and $\xi_{i}^{*}$ are introduced to represent the distance from actual values to the corresponding boundary values of the $\varepsilon$-tube. Then, Eq.(2) is transformed into the following constrained form:

$$
\begin{gathered}
\text { Min } \mathrm{S}=\frac{1}{2}\|\omega\|^{2}+C \sum_{i=1}^{n}\left(\xi_{i}+\xi_{i}^{*}\right) \\
\text { s.t. }\left\{\begin{array}{c}
d_{i}-\omega \cdot \varphi\left(x_{i}\right)-b \leq \varepsilon+\xi_{i}, \\
\omega \cdot \varphi\left(x_{i}\right)+b-d_{i} \leq \varepsilon+\xi_{i}^{*}, \\
\xi_{i}, \xi_{i}^{*} \geq 0,
\end{array}\right.
\end{gathered}
$$

Introduction Lagrange multipliers $\alpha_{i}, \alpha_{i}^{*}, \eta_{i}$ and $\eta_{i}^{*}$, we can write the corresponding primal Lagrangian as:

$$
\begin{aligned}
& L\left(\omega, \xi_{i}, \xi_{i}^{*}\right)=\frac{1}{2}\|\omega\|^{2}+C \sum_{i=1}^{n}\left(\xi_{i}+\xi_{i}^{*}\right) \\
& -\sum_{i=1}^{n} \alpha_{i}\left(\varepsilon+\xi_{i}+d_{i}-\omega \cdot \varphi\left(x_{i}\right)-b\right) \\
& -\sum_{i=1}^{n} \alpha_{i}^{*}\left(\varepsilon+\xi_{i}+\omega \cdot \varphi\left(x_{i}\right)+b-d_{i}\right) \\
& -\sum_{i=1}^{n}\left(\eta_{i} \xi_{i}+\eta_{i}^{*} \xi_{i}^{*}\right)
\end{aligned}
$$


Finally, by applying Karush-Kuhn-Tucker (KKT) conditions for regression, the optimization formulation can be transformed into a dual problem, get:

$$
\begin{aligned}
\operatorname{Max} \mathrm{Q}\left(\alpha_{i}, \alpha_{i}^{*}\right)=\sum_{i=1}^{n} y_{i}\left(\alpha_{i}^{*}-\alpha_{i}\right)-\varepsilon \sum_{i=1}^{n}\left(\alpha_{i}^{*}+\alpha_{i}\right)-\frac{1}{2} \sum_{i, j=1}^{n}\left(\alpha_{i}^{*}-\alpha_{i}\right)\left(\alpha_{j}^{*}-\alpha_{j}\right) K\left(x_{i}, x_{j}\right) \\
\text { s.t. }\left\{\begin{array}{l}
\sum_{i=1}^{n}\left(\alpha_{i}^{*}-\alpha_{i}\right)=0, \\
0 \leq \alpha_{i}, \alpha_{i}^{*} \leq C, i=1,2, \cdots, n
\end{array}\right.
\end{aligned}
$$

where $\alpha_{i}, \alpha_{i}^{*}$ are the so-called Lagrangian multipliers. They satisfy the equalities $\alpha_{i} \cdot \alpha_{i}^{*}=0$.

The Lagrange multipliers $\alpha_{i}$ and $\alpha_{i}^{*}$, are calculated and an optimal weight vector of the regression hyperplane is expressed as:

$$
w^{*}=\sum_{i=1}^{n s v}\left(\alpha_{i}-\alpha_{i}^{*}\right) K\left(x_{i}, x\right)
$$

Hence, the regression function is expressed as

$$
f(x)=\sum_{i=1}^{n s v}\left(\alpha_{i}-\alpha_{i}^{*}\right) K\left(x_{i}, x\right)+b
$$

Where the constant $b$ is written as

$$
b=\frac{1}{2} \sum_{i=1}^{n s v}\left(\alpha_{i}-\alpha_{i}^{*}\right)\left(K\left(x_{r}, x_{i}\right)+K\left(x_{s}, x_{i}\right)\right)
$$

Notably, a number of coefficients $\left(\alpha_{i}-\alpha_{i}^{*}\right)$ are non-zero values and the corresponding training data points have approximation error equal to or larger than zero. They are called support vectors. $x_{r}$ and $x_{s}$ in Eq.(9) are support vectors. $n s v$ denotes the number of the total support vectors.

The term $K\left(x_{i}, x_{j}\right)$ in Eq.(8) is defined as kernel function, where the value of kernel function equals the inner product of two vectors $x_{i}$ and $x_{j}$ in the feature space $\varphi\left(x_{i}\right)$ and $\varphi\left(x_{j}\right)$, meaning that $K\left(x_{i}, x_{j}\right)=\varphi\left(x_{i}\right) \varphi\left(x_{j}\right)$. The kernel function is intended to handle any dimension feature space without the need to calculate $\varphi(x)$ accurately. If any function can satisfy the Mercer's conditions and performs the non-linear mapping. In SVM, radial basis function (RBF) $K\left(x_{i}, x_{j}\right)=\exp \left(-\left\|x_{i}-x_{j}\right\| / 2 \sigma^{2}\right)$, polynomial basis function $K\left(x_{i}, x_{j}\right)=\left(\left(x_{i} \cdot x_{j}\right)+b\right)^{d}(b \geq 0, d$ is the natural number), and sigmoid function $K\left(x_{i}, x_{j}\right)=\tanh \left(k\left(x_{i} \cdot x_{j}\right)+v\right)$ are typical examples of kernel function. Only one variable needs to be determined in the radial basis function, thus fewer free parameters in SVM are needed to be determined using the radial basis function, which avails to optimize parameters. In addition, SVM constructed by radial basis function has excellent nonlinear classification ability. Thus, in this work, RBF is used in the SVM. Here, $C$ and $\sigma$ are user-determined free parameters, the selection of the parameters plays an important role in the performance of SVM.

\section{SVR-GA based prediction model}

We propose real-value genetic algorithms (RGA) to determine free parameters of SVR, known as GA-SVR, which optimizes all SVR's parameters simultaneously from the training data. By learning and training, we use the data of this subset to find interrelationship of input and output and get the solution by the SVR model.

The hybrid GA-SVR Model development and implementation for forecasting building cooling load is given as follows.

\section{A. Parameters analysis in SVR}

In SVR, parameters inappropriately selection may result in over-fitting or under-fitting problem which influence the performance of model. To construct the SVR model efficiently, SVR's parameters must be set carefully. Different parameter settings can cause significant differences in performance. Therefore, selecting the optimal hyper-parameter is an important step in SVR design. 
SVR generalization performance and efficiency depends on the hyper-parameters $(C, \varepsilon$ and kernel parameters $\sigma^{2}$ ), being set correctly. we must notice that each of them reaching an optimal point sometimes does not lead to a good performance for SVR, so just when the combination of them arrives an optimal value, we may get a nice performance.

Genetic algorithms are optimization and search technique philosophically based on the concepts of biological evolution (natural genetics and natural selection) and Darwin's theory of survival of the best. These algorithms are used to solve linear and nonlinear problems by exploring all regions of state space and exploiting potential areas through mutation, crossover and selective operations applied to individuals in the population [6]. The advantage of GAs is that they give the designer a family of near optimal designs with a small variation in performance index instead of a single solution.

The generalization performance of SVR and its efficiency are dependent upon the hyper-parameters $(C, \varepsilon$ and kernel parameters $\sigma)$ to be set correctly. The grid search algorithm is an alternative to finding the best $\mathrm{C}$ and $\sigma$. However it is time consuming and can not converge at the global optimum. Some authors have proposed selecting parameters using cross-validation, but this method is very computationally intensive and data-intensive. Therefore, we adopt RGA to seek the optimal parameters of SVR in order to improve the efficiency of prediction.

In addition, the problem existing in the binary coding lies in the fact that a long string always occupies the computer memory even though only a few bits are actually involved in the crossover and mutation operation. This is particularly the case when a lot of parameters are needed to be adjusted in the same problem and a higher precision is required for the final result. This is also the major problem while initialing values of parameters of SVM in advance. To overcome the inefficient occupation of the computer memory, the underlying real-valued crossover and mutation algorithm are employed. Different from the binary genetic algorithm (BGA), the real-valued genetic algorithm (RGA) uses real value as a parameter of the chromosome in populations without coding and encoding process before calculating fitness value. Consequently, the RGA is more straightforward, faster and efficient than the BGA.

\section{B. GA-SVR model}

This model adopts RGA to seek the optimal values of SVR's parameters and improve the prediction efficiency. The proposed GA-SVR model dynamically optimizes the values of SVR's parameters through RGA evolutionary process, and using acquired parameters to construct optimized SVR model in order for proceeded prediction. RGA attempts to search for optimal parameters which allow SVR to fit various data sets. Fig. 1 illustrates the algorithm process of the GA-SVR model

(1) Chromosome representation and population size

Unlike traditional BGA, in RGA for optimization problems, all of the corresponding parameters or variables are directly coded to form a chromosome.Therefore, the representation of the chromosome is straightforward in RGA. The three SVR parameters $\sigma^{2}, C$ and $\varepsilon$ were directly coded to generate the chromosome in the present method. The chromosome $x$ was represented as $x=\left\{p_{1}\right.$, $\left.p_{2}, p_{3}\right\}$, where $p_{1}, p_{2}$ and $p_{3}$ denote the regularization parameters $\sigma^{2}, C$ and $\varepsilon$, respectively. The initial population size is composed of 150 randomly created chromosomes. 
(2) The definition and calculation of fitness function

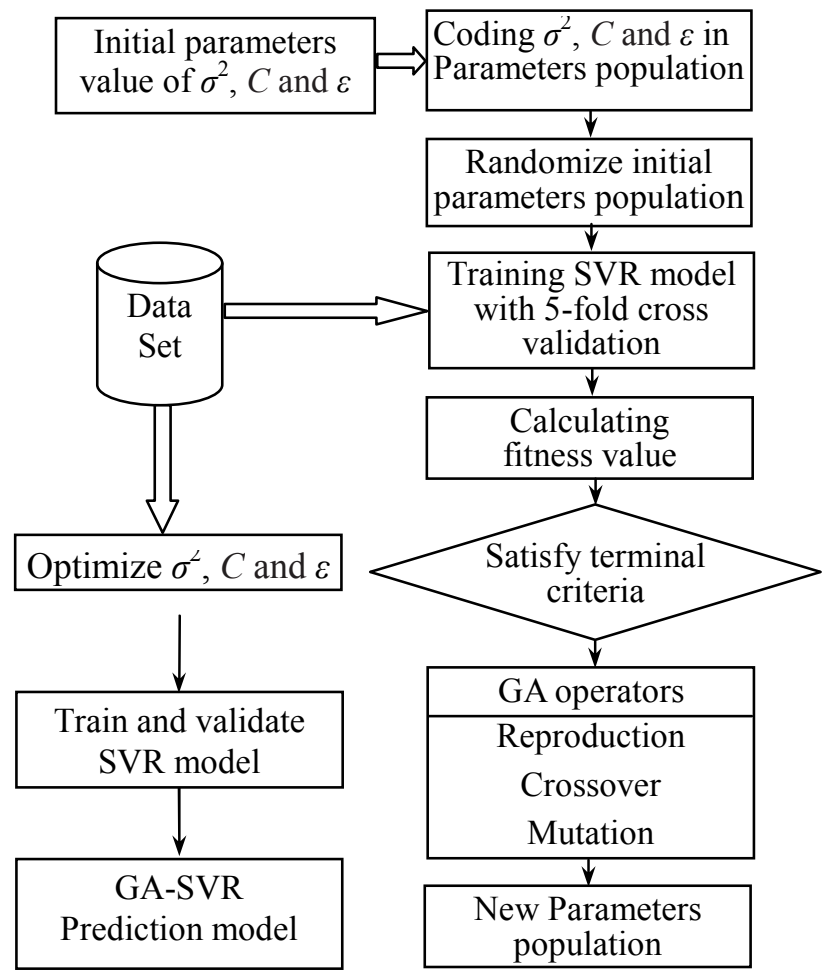

Fig.1 GA-SVR prediction model

A fitness function must be designed to determine the fitness of each individual solution before starts to search optimal values of SVR parameters. Several measurement indicators have been proposed and used to evaluate the prediction accuracy of model such as MAPE, RMSE, and maximum error in time series prediction problems. In this study, the 5-fold leave-one-out cross-validation method is used to evaluate fitness. In 5-fold cross validation, the training data set is randomly divided into 5 mutually exclusive subsets of approximately equal size. The regression function is built with a given set of parameters $\left\{\sigma^{2}, C\right.$ and $\left.\varepsilon\right\}$, using 4 subsets as the training set. The performance of the parameter set is evaluated by the MAPE on the last subset. The above procedure is repeated 5 times, so that each subset is used once for testing. Averaging the MAPE over the 5 trials gives an estimate of the expected generalization error. Thus, the fitness function is defined as follows:

$$
\begin{aligned}
\text { fitness } & =\min \text { MAPE } \\
& =\min \frac{1}{\mathrm{M}} \sum_{i=1}^{M}\left|\frac{y_{k}-\hat{y}_{k}}{y_{k}}\right| \times 100 \%
\end{aligned}
$$

Where $y_{k}$ and $\hat{y}_{k}$ represent the actual and validation values respectively, $M$ is the number of training samples.

(3) Genetic operators

The real-valued genetic algorithm uses selection, crossover, and mutation operators to generate the offspring of the existing population. Selection is performed to select excellent chromosomes to reproduce. Based on fitness function, chromosomes with higher fitness values are more likely to yield offspring in the next generation by means of the roulette wheel. The chromosomes that survive in the next generation then are placed in a mating pool for the crossover and mutation operations. The arithmetic crossover is applied to randomly paired chromosome and produces two complimentary linear combinations of the parents. The mutation operation follows the crossover operation and determines whether a chromosome should be mutated in the next generation. This study applied non-uniform mutation method. Offspring replaces the old population and forms a new population in the next generation by the three operators, the evolutionary process proceeds until stop conditions are satisfied. 


\section{Experiment principle and dataset}

\section{A. Experiment measure principle}

In this paper, the fundamental of forward-voltage based method is used to measure the junction temperature and the thermal resistance of power LED.

Thermail resistance $R_{t h}$ is the ratio of the changes of temperature and thermal dissipated power, it can be expressed as:

$$
R_{t h}=\frac{T_{j}-T_{0}}{P}
$$

Where $T_{j}$ is the junctino temperature, $T_{0}$ is the environment temperature and $P$ is the thermal dissipated power of LED.

At low current, the changes of junction temperature $\Delta T_{j}$ and forward voltage drop $\Delta V_{F}$ show a good linear relationship. The $\Delta T_{j}$ can be expressed as:

$$
\Delta T_{j}=T_{j}-T_{0}=K\left(V_{t}-V_{0}\right)=K \Delta V_{F}
$$

Where $K$ is the temperature sensivity parameter(TSP) which is also call the $K$ coefficient. It can be determined by measuring the voltage drop across the LED juntion at two known ambient temperature, in other word, $K$ can be expressed as:

$$
K=\left|\frac{V_{H}-V_{L}}{T_{H}-T_{L}}\right| m V /{ }^{\circ} \mathrm{C}
$$

Where $V_{H}$ and $V_{L}$ are forward potentials at two known ambient temperatures, $T_{H}$ and $T_{L}$, respectively.

\section{B. Experimental setup}

An electronic circuit was built to create the necessary current pulses to measure the voltage across the junction. Using the equation described above, the junction temperature of high-power LEDs was estimated. Fig. 2 is a schematic of the experimental setup. The forward-voltage method used in this study needs electric circuits that can provide the function of pulsed current. The high accurate data acquisition card was used to monitor the forward voltage.

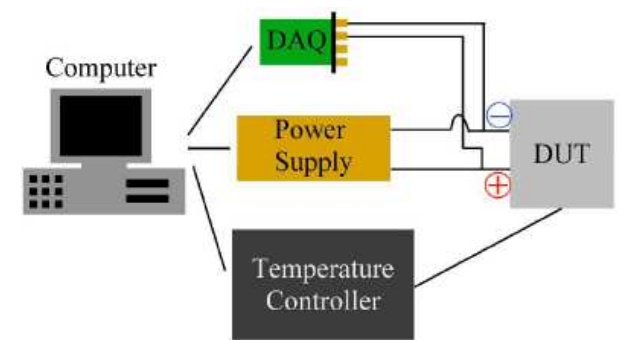

Fig.2 Forward-voltage schematic of the experimental setup

All the measurement processes were programmable. In this study, the thermal resistance of junction-to-case Rjc was investigated. The LED was placed inside the thermal chamber to keep it at a constant ambient temperature. The temperature inside the thermal chamber was controlled by a heater controller, which received temperature information from the resistance temperature detector and turned the heater on and off. To measure $K$-factor, the forward voltage drop across junction at two different case temperatures should be measured. The forward voltage of LED decreased when the junction temperature increased.

\section{Dataset and evaluation indices}

In this study, GA-SVR was applied to model and predict the thermal resistance. The measured data of high power LED thermal resistance are divided into two parts and these parts constitute training and testing data. The experimental data set consists of 50 values. The 45 values are used for training of SVR with GA whereas, the 5 values are performed for testing of the GA-SVR prediction model performance. 
To evaluate its adaptability to different predict methods, three error indicators: Relative Mean Errors (RME), Mean Absolute Relative Error (MARE) and Root Mean Squared Errors (RMSE) are applied as performance indices.

$$
\begin{gathered}
R M E=\frac{1}{N} \sum_{i=1}^{N} \frac{y_{i}-y_{i}^{*}}{y_{i}} \\
M A R E=\frac{1}{N} \sum_{i=1}^{N} \frac{\left|y_{i}-y_{i}^{*}\right|}{y_{i}} \\
R M S E=\sqrt{\frac{1}{N} \sum_{i=1}^{N}\left(\frac{y_{i}-y_{i}^{*}}{y_{i}}\right)^{2}}
\end{gathered}
$$

Where $y_{i}$ is the actual value and $y_{i}{ }^{*}$ is the predicted value.

\section{Results and dicussions}

Fig. 3 shows the comparison of measured and estimated thermal resistance by using GA-SVR and BPNN model, respectively at constant power when the ambient temperature changes from $25^{\circ} \mathrm{C}$ to $70^{\circ} \mathrm{C}$. Most of points by using GA-SVR are more close to the experimental values than those of BPNN model. This reflects that, by comparison with the BPNN model, GA-SVR model gives the most satisfactory agreement with the experimental values.

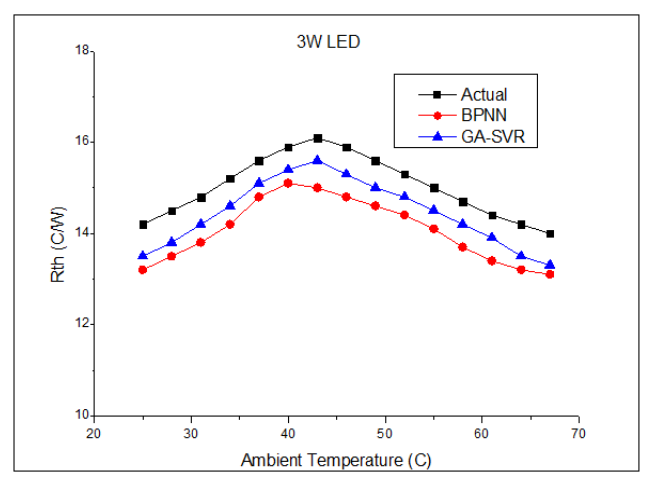

(a)

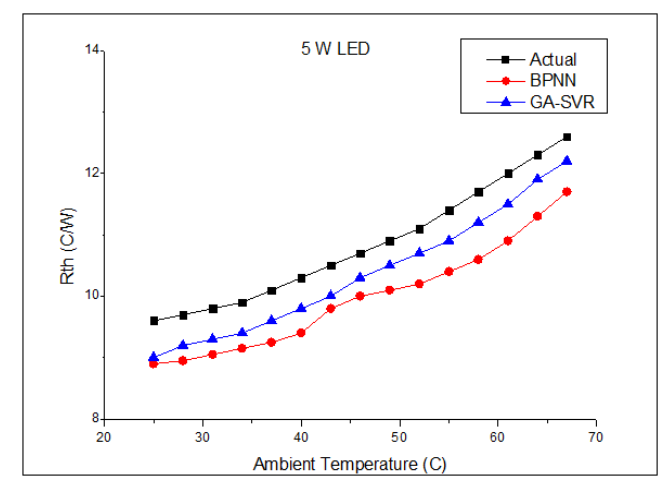

(b)

Fig.3 Comparison of experimental values vs. estimated values predicted by GA-SVR and BPNN model

From Tab.1, it can be observed that, all other samples' absolute percentage errors predicted by GA-SVR are smaller than those of the BPNN model. In generalized performance, the GA-SVR predictor is also superior to the traditional SVR. This means that GA-SVR is the most excellent predictor.

Tab. 1 Evaluation Indices comparison GA-SVR vs BPNN

\begin{tabular}{|c|c|c|c|}
\hline \multirow{2}{*}{ Predictor } & \multicolumn{3}{|c|}{ Evaluation Indices } \\
\cline { 2 - 4 } & RME\% & MARE\% & RMSE\% \\
\hline BPNN & 0.6145 & 2.615 & 4.815 \\
\hline GA-SVR & 0.4015 & 1.146 & 2.717 \\
\hline
\end{tabular}

From Fig.3, it is also observed that there is no significant difference in thermal resistance for $3 \mathrm{~W}$ LEDs when the ambient temperature increases. However, for 5W LED the thermal resistance from junction to board increases with ambient temperature for a given heat sink. 


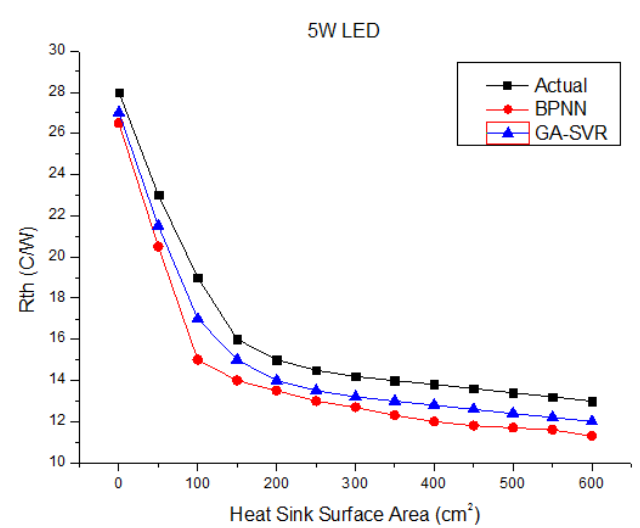

Fig.4 Thermal resistance comparison with different heat sink surface area

Fig. 4 shows the thermal resistance from junction to board decreases for 5W LED with the surface area of the heat sink increase. This reveals that addition of the heat sink surface area can efficiently decrease the thermal resistance of high power LED.

\section{Conclusions}

The equipment for LEDs thermal resistance measurement was developed and applied to study the thermal performance of high power LEDs. A forward-voltage method was utilized in this study. Then SVR is introduced to forecast high power LED thermal resistance under difference operation conditions. In this study, a new GA-SVR, in which the hyper-parameters of support vector regression model were chosen by genetic algorithm, was proposed for predicting the LED thermal resistance with very good predictive accuracy. In order to demonstrate the superiority of the SVR model, a BPNN model was also designed. The comparative study showed that the modeling errors presented by the SVR model was much smaller that those given by the BPNN model. The superior performance of the GA-SVR model is firstly due to the generalization ability of SVR model for forecasting and the proper selection of SVR parameters by GA. In addition, an decreasing trend of $\mathrm{Rj}$, with respect to larger heat sink surface area of 5W LED was also reported.

\section{Acknowledgments}

This work is partly supported by the National Natural Science Foundation of China (50905119); the Natural Science Foundation of Guangdong Province (941806001001985); China Postdoctoral Science Foundation special fund (201104354); Shenzhen Science \& Technology Program (SYB200907090130A / JSA200903190981A) and the SZU R/D Fund (2010004).

\section{Reference}

[1] Weng C J. Advanced thermal enhancement and management of LED packages. International Communications in Heat and Mass Transfer, 2009: 245

[2] Gao S, Hong J. Design optimization on the heat transfer and mechanical reliability of high brightness light emitting diodes (HBLED) package. Electronic Components and Technology Conference, 2008: 798

[3] Chhajed, S., Yangang Xi and Gessmann, T., Proceedings of the SPIE-The International Society for Optical Engineering, v5739, n1, p.16-24 (2005).

[4] Xi, Yangang, Gessmann, Thomas and Xi, Jingqun, Japanese Journal of Applied Physics, Part 1: Regular Papers and Short Notes and Review Papers, v44, n10, p.7260-7266, October 11 (2005).

[5] Chin C.Lee, Temperature Measurement of Visible Light-Emitting Diodes Using Nematic Liquid Crystal ThermographyWith Laser Illumination, IEEE Photonics Technology Letters, Vol.16, NO.7, p.1706 (2004).

[6]G.E. Goldberg, Genetic Algorithms in Search, Optimization and Machine Learning, Addison-Wesley, NY, USA, 1989. 